\title{
Investigating the role and mechanism of microRNA-196a in oral squamous cell carcinoma by targeting FOXO1
}

\author{
HONGNING SONG ${ }^{1,2}$, LINFENG LAI ${ }^{3}$, MIN LIU $^{2}$, XUXIA WANG ${ }^{1}$, JUN ZHANG $^{4}$ and SHANYONG ZHANG ${ }^{1,5}$ \\ ${ }^{1}$ Department of Oral and Maxillofacial Surgery, School and Hospital of Stomatology, Shandong University \\ and Shandong Provincial Key Laboratory of Oral Tissue Regeneration and Shandong Engineering Laboratory \\ for Dental Materials and Oral Tissue Regeneration, Jinan, Shandong 250012; ${ }^{2}$ Department of Stomatology, \\ The Second Affiliated Hospital of Shandong First Medical University, Taian 271000; ${ }^{3}$ Department of Oral and \\ Maxillofacial Surgery, Wenzhou Central Hospital, Wenzhou, Zhejiang $325000 ;{ }^{4}$ Department of Orthodontics, \\ School and Hospital of Stomatology, Shandong University and Shandong Provincial Key Laboratory of \\ Oral Tissue Regeneration and Shandong Engineering Laboratory for Dental Materials and Oral Tissue \\ Regeneration, Jinan, Shandong 250012; ${ }^{5}$ Department of Oral and Maxillofacial Surgery, School of Medicine, \\ Ninth People's Hospital, Shanghai Jiao Tong University, Shanghai 200011, P.R. China
}

Received November 29, 2018; Accepted January 6, 2020

DOI: $10.3892 / \mathrm{etm} .2020 .8614$

\begin{abstract}
Oral squamous cell carcinoma (OSCC) is one of the most prevalent malignancies worldwide. MicroRNAs (miRNAs or miRs) serve crucial roles in the development of OSCC. miR-196a is upregulated in various tumors; however, the role of miR-196a in OSCC remains unclear. This present study aimed to determine the role and underlying mechanism of miR-196a in OSCC cells. Reverse transcription-quantitative PCR (RT-qPCR) was used to measure miR-196a levels in OSCC cells. MTT assays were also performed to determine cell proliferation. Cell migration was detected using wound healing assays and transwell assays, and cell apoptosis was analyzed via flow cytometry. The results indicated that the expression of miR-196a was increased in OSCC cells compared with normal oral squamous cells. TargetScan and luciferase reporter assays also confirmed that forkhead box $\mathrm{O} 1$ (FOXO1) was a target gene of miR-196a. It was demonstrated that FOXO1 small interfering RNA significantly promoted SCC9 cell proliferation and migration, and inhibited cell apoptosis. Furthermore, inhibition of miR-196a suppressed SCC9 cell proliferation and migration, and induced cell apoptosis. However, all effects of the miR-196a inhibitor
\end{abstract}

Correspondence to: Dr Shanyong Zhang, Department of Oral and Maxillofacial Surgery, School and Hospital of Stomatology, Shandong University and Shandong Provincial Key Laboratory of Oral Tissue Regeneration and Shandong Engineering Laboratory for Dental Materials and Oral Tissue Regeneration, 44-1 Wenhua Road West, Jinan, Shandong 250012, P.R. China

E-mail: zhangsy987456@163.com

Key words: oral squamous cell carcinoma, microRNA-196a, forkhead box $\mathrm{O} 1$ were reversed following FOXO1 inhibition. Western blotting and RT-qPCR were subsequently performed to determine the effect of miR-196a on the PI3K/Akt signaling pathway. In the present study, transfection of miR-196a inhibitor suppressed the expression of phosphorylated (p)-PI3K and p-Akt, and enhanced the levels of FOXO1, while inhibition of FOXO1 exerted the opposite effects. Furthermore, it was demonstrated that miR-196a mimic significantly enhanced SCC9 cell proliferation and migration, and inhibited cell apoptosis. In conclusion, the results indicated that miR-196a serve as an oncogene in OSCCs. Downregulation of miR-196a inhibited the malignant biological processes of OSCC cells by targeting FOXO1. The current results may provide a novel therapeutic strategy for the treatment of patients with OSCC.

\section{Introduction}

Oral cancer is one of the most common malignancies of the head and neck, with an incidence rate that is increasing $(1,2)$. Oral and oropharyngeal squamous cell carcinomas (OSCCs) accounted for $90 \%$ of oral cancer incidence in $2012(3,4)$. Several therapeutic technologies including radiotherapy, chemotherapy, traditional surgery and targeted therapy, have been utilized as major viable treatments for OSCC. However, patients with OSCC generally have a poor prognosis and the 5 -year survival rate has not improved significantly in the last 10 years (5). OSCC has a poor prognosis due to its low response rate to drug-based therapy (3). Dysregulation of relative signaling cascades may result in tumorigenesis and cancer resistance to clinical treatment. Therefore, identifying the molecular mechanisms of OSCC development and progression is essential for the development of novel clinical therapies to improve the survival rate of patients with OSCC.

MicroRNAs (miRNAs or miRs) are a group of non-coding RNAs, which consist of 20-22 nucleotides (6). miRNAs mediate gene expression by binding to the 3 ' untranslated region (UTR) 
of target mRNAs (7-9). miRNAs serve critical roles in the normal development of various biological processes, including cellular differentiation, cell proliferation and cell apoptosis (10). Studies have indicated that miR-196a expression is up-regulated in various types of cancer and its expression is closely associated with a poor survival rate (11-14). Similarly, a number of studies have indicated that miR-196a may promote the proliferation and cellular invasion of various types of cancer, including non-small cell lung cancer, gastric cancer and breast cancer (11-14). Furthermore, miR-196a has been reported to function by targeting forkhead box $\mathrm{O} 1$ (FOXO1) in lung, human liver and cervical cancer cells (15-17). However, the expression and mechanisms of miR-196a action in OSCCs are not well established. Previous studies have demonstrated that the PI3K/Akt pathway is activated in most types of cancer and suppresses the function of FOXO genes (18-22). The expression of FOXO1 is upregulated in human OSCCs and is significantly correlated with the advancement of clinical stages $(23,24)$. However, the expression profile and underlying mechanisms of FOXO1 in OSCC remain unknown.

Therefore, the present study aimed to investigate the role of miR-196a and FOXO1 in OSCC cells and to further explore its underlying mechanisms of action.

\section{Materials and methods}

Cell culture. The human OSCC (SCC9) and primary normal human oral keratinocyte (HOK) cell lines were obtained from American Type Culture Collection. Cells were maintained in RPMI 1640 medium (Invitrogen; Thermo Fisher Scientific, Inc.) containing 10\% FBS (Invitrogen; Thermo Fisher Scientific, Inc.) and $1 \%$ penicillin-streptomycin solution (Sigma-Aldrich; Merck KGaA). Cells were incubated at $37^{\circ} \mathrm{C}$ with $5 \% \mathrm{CO}_{2}$.

Plasmids and cell transfection. miR-196a mimic, mimic control, miR-196a inhibitor or the negative control of miR-196a inhibitor (inhibitor-NC) were purchased from Guangzhou RiboBio Co., Ltd. Control-small interfering RNA (siRNA; cat. no. sc-36869) and FOXO1-siRNA (cat. no. sc-35382) were purchased from Santa Cruz Biotechnology, Inc. SCC9 cells, which were seeded into six-well plates $\left(5 \times 10^{4}\right.$ per well), at the exponential growth phase were cultured at $37^{\circ} \mathrm{C}$ for $24 \mathrm{~h}$ in RPMI 1640 medium containing $10 \%$ FBS and then transfected with miR-196a inhibitor, Inhibitor-NC, control-siRNA, FOXO1-siRNA, or miR-196a inhibitor+ FOXO1-siRNA using Lipofectamine ${ }^{\circledR} 2000$ reagent (Invitrogen; Thermo Fisher Scientific, Inc.) according to the manufacturer's protocol. Cells without any treatment were considered controls. After incubation at $37^{\circ} \mathrm{C}$ for $48 \mathrm{~h}$, transfection efficiency was detected using RT-qPCR.

Cell viability analysis. An MTT assay was used to detect SCC9 cell proliferation. $5 \times 10^{3}$ cells were seeded in 96-well plate (BD Biosciences) at $37^{\circ} \mathrm{C}$ for $24 \mathrm{~h}$. Subsequently, SCC9 cells were transfected as previously described. Cells were then inhibited at $37^{\circ} \mathrm{C}$ for 12,24 or $48 \mathrm{~h}$. MTT reagent $(10 \mu \mathrm{l}$; cat. no. C0009; Beyotime Institute of Biotechnology) was added to each well and incubated for another $4 \mathrm{~h}$ at $37^{\circ} \mathrm{C}$. DMSO (100 $\mu \mathrm{l}$; Nanjing KeyGen Biotech Co., Ltd.) was used to dissolve the resulting formazan crystals. Absorbance at $490 \mathrm{~nm}$ was then analyzed using a microplate reader (Omega Bio-Tek). The experiment was repeated in triplicate.

Cell apoptosis analysis. SCC9 cells were transfected with miR-196a mimic, mimic control, miR-196a inhibitor, inhibitor-NC, control-siRNA, FOXO1-siRNA, or miR-196a inhibitor + FOXO1-siRNA as previously described. After transfection, cell apoptosis was analyzed using an Annexin V-FITC/propidium iodide Apoptosis Detection kit (BD Biosciences) according to the manufacturer's protocol. An FC500 flow cytometer (Beckman Coulter, Inc.) was used to measure the extent of apoptosis. Each experiment was repeated in triplicate and CellQuest software version 5.1 (Becton, Dickinson and Company) was used to analyze the rate of apoptosis.

Transwell migration assay. SCC9 cells were transfected with miR-196a mimic, mimic control, miR-196a inhibitor, Inhibitor-NC, control-siRNA, FOXO1-siRNA, or miR-196a inhibitor+ FOXO1-siRNA as previously described and cultured in serum free RPMI 1640 medium at $37^{\circ} \mathrm{C}$ for $48 \mathrm{~h}$. Cells were harvested using trypsin (Gibco; Thermo Fisher Scientific, Inc.), centrifuged $\left(1,000 \times \mathrm{g} ; 5 \mathrm{~min} ; 4^{\circ} \mathrm{C}\right)$ and re-suspended in RPMI 1640 medium containing $0.1 \%$ FBS. Subsequently, cells $\left(1 \times 10^{4}\right)$ were added to the upper chamber of a transwell apparatus $(8 \mu \mathrm{m}$ pore size; Corning Inc.), while RPMI 1640 culture medium with $10 \%$ FBS was added to the lower culture chamber. Cells were then cultured at $37^{\circ} \mathrm{C}$ with $5 \% \mathrm{CO}_{2}$ for $48 \mathrm{~h}$. Cells on the underside of membranes were washed with cold PBS three times, fixed with $4 \%$ paraformaldehyde, at room temperature for $30 \mathrm{~min}$ and stained for $10 \mathrm{~min}$ at room temperature with $0.1 \%$ crystal violet. The lower membrane cells were counted from 5 random areas using an inverted light microscope (Olympus IX51; x100 magnification; Olympus Corporation).

Wound-healing assay. To investigate cell migration, wound healing assays were performed. At $48 \mathrm{~h}$ following transfection, transfected SCC9 cells were seeded in 24-well plates $\left(5 \times 10^{5}\right.$ cells $\left./ \mathrm{ml}\right)$ and cultured at $37^{\circ} \mathrm{C}$ for $24 \mathrm{~h}$, allowing $80 \%$ confluency to be reached. Cells were scratched using a $10 \mu \mathrm{l}$ pipette tip and the debris were removed with PBS. After a second incubation in serum-free RPMI 1640 medium at $37^{\circ} \mathrm{C}$ for $24 \mathrm{~h}$, cells were washed to remove culture medium and representative images were captured at x100 magnification using an inverted light microscope. Wound areas were recorded and analyzed using Image-Pro Plus 6.0 software (National Institute of Health). The size of the wound was measured compared with the original wound following $24 \mathrm{~h}$. Percent of wound $(\%)=$ wound width at $24 \mathrm{~h} /$ wound width at $0 \mathrm{~h} \times 100 \%$. Each experiment was performed in triplicate.

Reverse transcription-quantitative PCR (RT-qPCR). After experimental treatments, total RNA from SCC9 cells and HOK cells was extracted from cells using TRIzol ${ }^{\circledR}$ reagent (Invitrogen; Thermo Fisher Scientific, Inc.) according to the manufacturer's protocol. U6 was used as the endogenous control for miRNA expression and GAPDH was used as the endogenous control to normalize mRNA. The PrimeScript ${ }^{\mathrm{TM}}$ RT reagent kit (Takara Bio, Inc.) was used for cDNA syntheses, and the temperature protocol was as follows: $70^{\circ} \mathrm{C}$ for $5 \mathrm{~min}$, 
$37^{\circ} \mathrm{C}$ for $5 \mathrm{~min}$ and $42^{\circ} \mathrm{C}$ for $1 \mathrm{~h}$. RT-qPCR was performed using SYBR Premix Ex Taq (Takara Bio Inc.) according to the manufacturer's protocol. RT-qPCR was performed as follows: $95^{\circ} \mathrm{C}$ for $10 \mathrm{~min}$, followed by 40 cycles of $15 \mathrm{sec}$ at $95^{\circ} \mathrm{C}, 72^{\circ} \mathrm{C}$ for $30 \mathrm{sec}$ and a final dissociation stage $\left(78^{\circ} \mathrm{C}\right.$ for $\left.1.5 \mathrm{~min}\right)$. Relative gene expression was determined using the $2^{-\Delta \Delta \mathrm{Cq}}$ method (25). All experiments were performed in triplicate.

Western blot assay. After experimental treatments, total protein was extracted from OSCC cells using RIPA buffer (Auragene Bioscience Co.). BCA assay kit (Thermo Fisher Scientific, Inc.) was used to determine the concentration of protein samples. Proteins were resolved by $10 \%$ SDS-PAGE and then transferred onto PVDF membranes. After blocking with 5\% non-fat milk for $1 \mathrm{~h}$ at room temperature, membranes were incubated with the following primary antibodies overnight at $4{ }^{\circ} \mathrm{C}$ : PI3K (cat. no. 4257; 1:1,000; Cell Signaling Technology, Inc.), FOXO1 (cat. no. 2880; 1:1,000; Cell Signaling Technology, Inc.), Akt (cat. no. 9272; 1:1,000; Cell Signaling Technology, Inc.), phosphorylated (p)-Akt (cat. no. 9611; 1:1,000; Cell Signaling Technology, Inc.), p-PI3K (cat. no. 17366; 1:1,000; Cell Signaling Technology, Inc.), and GAPDH (cat. no. 5174; 1:1,000; Cell Signaling Technology, Inc.). Subsequently, membranes were washed three times with PBST and incubated with secondary antibody (cat. no. 7074; 1:2,000; Cell Signaling Technology, Inc.) for $2 \mathrm{~h}$ at room temperature. Immunoreactive protein bands were visualized using an ECL detection system (Bio-Rad Laboratories, Inc.) according to the manufacturer's protocol. Proteins were quantified using ImageJ version 1.49 software (National Institute of Health).

Dual luciferase reporter assay. The binding sites between miR-196a and FOXO1 were investigated using TargetScan bioinformatics software version 7.1 (www.targetscan.org/vert_71). To confirm direct target binding sites, the wild-type (WT) or mutant (Mut) FOXO1 genes were inserted into the Dual-luciferase miRNA Target Expression Vector (Guangzhou RiboBio Co., Ltd.). To point-mutate the miRNA-196a binding domain on the 3'UTR of FOXO1, the QuikChange Site-Directed Mutagenesis kit (Agilent Technologies, Inc.) was used following the manufacturer's protocol. The reporter vectors were then co-transfected with WT-FOXO1 or MUT-FOXO1 and miR-196a mimic or mimic control, into SCC9 cells using Lipofectamine ${ }^{\circledR} 2000$ reagent (Invitrogen; Thermo Fisher Scientific, Inc.) in accordance with the manufacturer's protocol. After culturing cells at $37^{\circ} \mathrm{C}$ for an additional $48 \mathrm{~h}, 100 \mu \mathrm{l}$ passive lysis buffer (Promega Corporation) was used to dissociate cells, after which luciferase activity was assessed using the dual-luciferase assay system (Promega Corporation) according to the manufacturer's protocol. All the experiments were performed in triplicate, and luciferase activity was normalized to Renilla luciferase activity.

Statistical analysis. All results were expressed as the mean \pm standard deviation. Statistical analysis was performed using Graphpad Prism 6 software (GraphPad Software, Inc.). Comparisons between two groups were assessed using Student's t-test, and comparisons between multiple groups were analyzed using one-way ANOVA followed by Tukey's post-hoc test. $\mathrm{P}<0.05$ was considered to indicate a statistically significant difference.

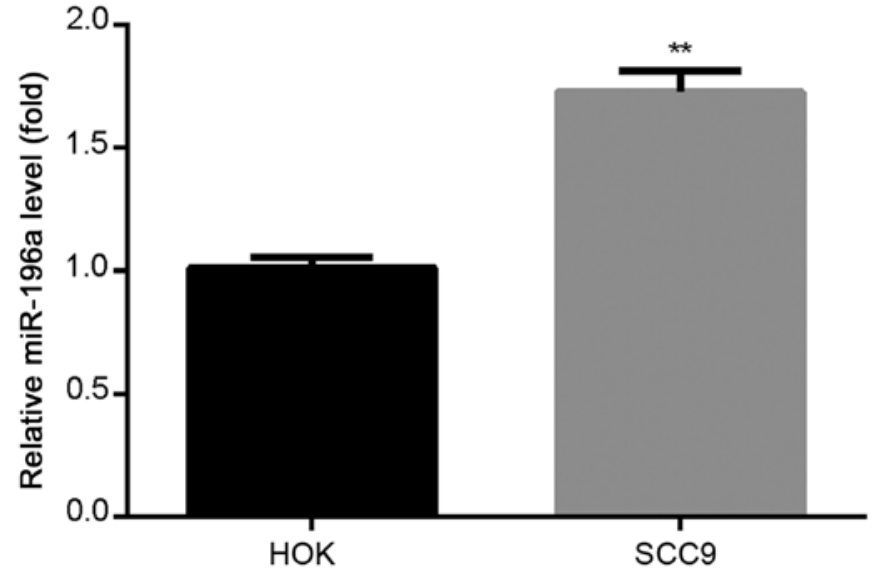

Figure 1. Expression of miR-196a in oropharyngeal squamous cell carcinoma cell lines. Reverse transcription-quantitative PCR was used to detect the expression of miR-196a in SCC9 cells and human oral keratinocyte cells. Data were expressed as the mean \pm standard deviation from three independent experiments. ${ }^{* *} \mathrm{P}<0.01$ vs. HOK cells. miR, microRNA.

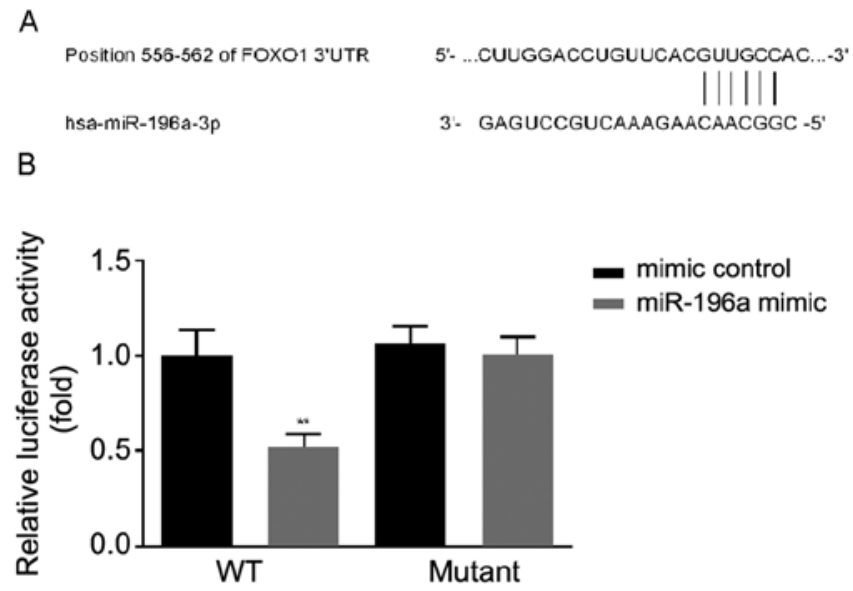

Figure 2. FOXO1 is a direct target of miR-196a. (A) binding sites between FOXO1 and miR-196a were elucidated using TargetScan. (B) Dual-luciferase analysis was performed to confirm the binding sites between FOXO1 and miR-196a. The results are expressed as the mean \pm standard deviation. ${ }^{* *} \mathrm{P}<0.01$ vs. mimic control. FOXO1, forkhead box O1; miR, microRNA; WT, wild-type.

\section{Results}

miR-196a expression is increased in OSCC cells. To evaluate the expression of miR-196a in human SCC9 cells and normal HOK cells, RT-qPCR was performed. The results revealed that the expression of miR-196a was significantly increased in SCC9 cells compared with HOK cells (Fig. 1).

FOXO1 is a target of miR-196a. miR-196a has been reported to function by targeting FOXO1 in lung, human liver and cervical cancer cells (15-17). To determine the molecular mechanisms by which miR-196a regulates the function of OSCC, the associations between FOXO1 and miR-196a in OSCC were assessed. According to TargetScan bioinformatics software analysis, binding sites between the 3' UTR of FOXO1 and miR-196a were identified (Fig. 2A). Subsequently, dual-luciferase assays were performed. The results revealed that compared with cells co-transfected with mimic control and FOXO1-WT, miR-196a 
A

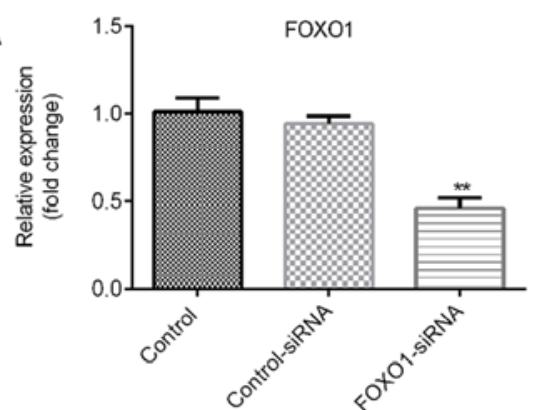

Control

$\infty 00$ Control-siRNA

$\square$ FOXO1-siRNA
B

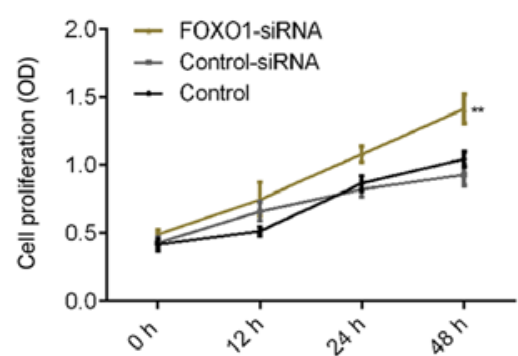

C
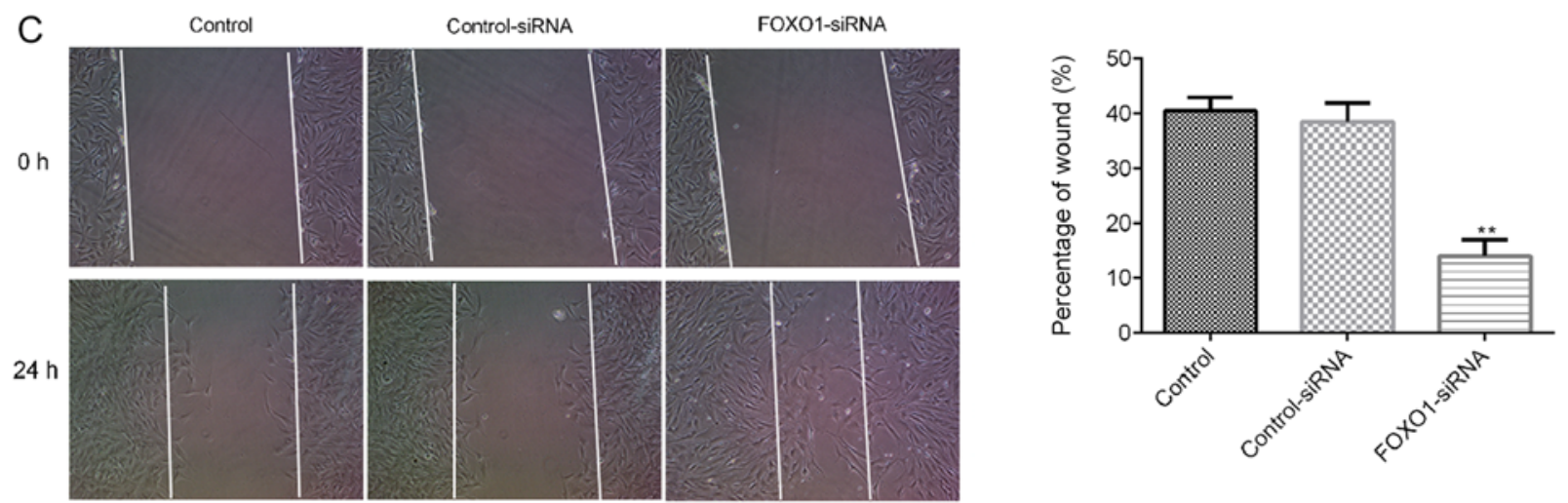

D
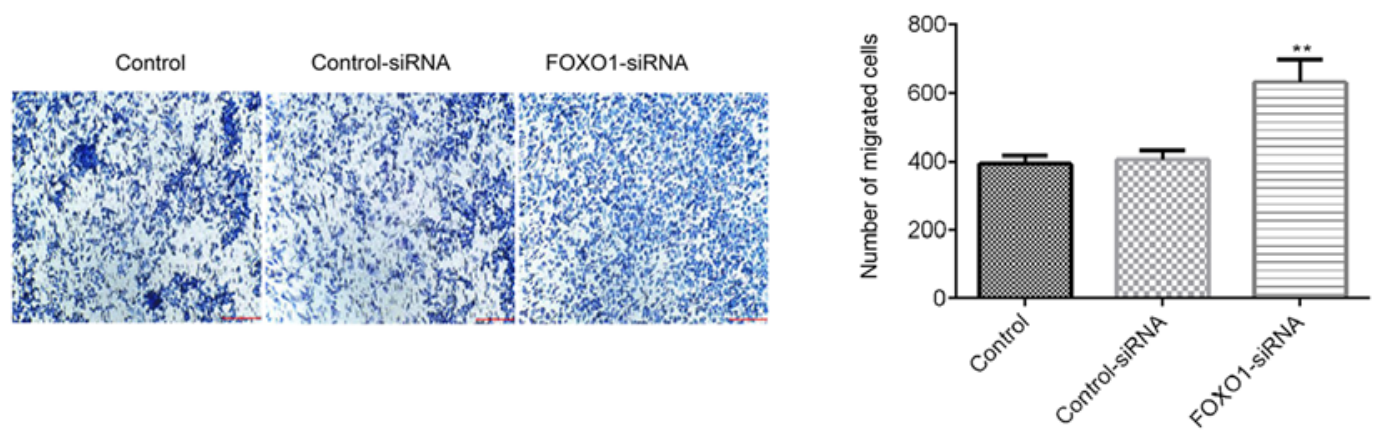

E
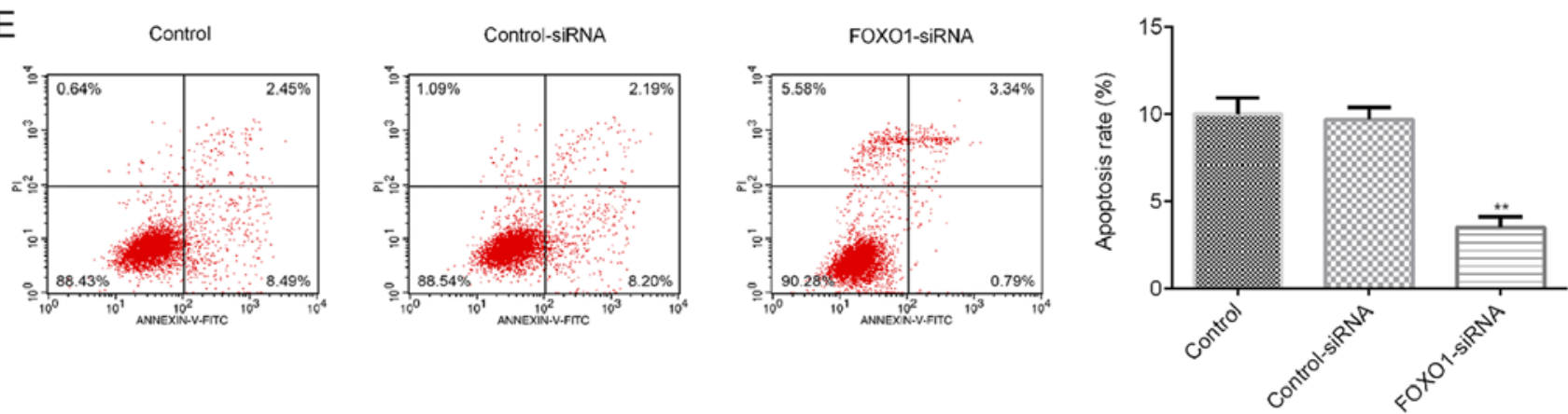

Figure 3. Effects of FOXO1-siRNA on SCC9 cells. SCC9 cells were transfected with control-siRNA or FOXO1-siRNA for 48 h. (A) Levels of miR-196a in SCC9 cells was detected via reverse transcription-quantitative PCR. (B) Cell proliferation was detected via MTT assays. (C) Cell migration was determined via wound healing assays and measuring the size of the wound compared with the original wound following $24 \mathrm{~h}$ (magnification, $\mathrm{x} 100$ ). (D) Cell invasion was analyzed with transwell assays (magnification, x100). (E) Cell apoptosis was analyzed via flow cytometry, and cell apoptosis rate was calculated as early apoptosis rate + late apoptosis rate. ${ }^{* *} \mathrm{P}<0.01$ vs. control-siRNA group. FOXO1, forkhead box O1; OD, optical density; siRNA, small interfering RNA.

significantly suppressed the luciferase activity of cells co-transfected with miR-196 mimic and FOXO1-WT. However, no significant changes were observed in cells co-transfected with miR-196 mimic and FOXO1-MUT (Fig. 2B). The results indicated that FOXO1 is a direct target of miR-196a.

Effect of FOXO1 silencing on OSCC cells. The effect of FOXO1 silencing on SCC9 cells was determined. SCC9 cells were transfected with control-siRNA or FOXO1-siRNA for $48 \mathrm{~h}$, after which the transfection efficiency was detected via RT-qPCR. As presented in Fig. 3A, when compared with the control-siRNA group, FOXO1-siRNA significantly reduced the mRNA levels of FOXO1 in SCC9 cells. Further analysis indicated that compared with the control-siRNA group, FOXO1-siRNA significantly increased the proliferation (Fig. 3B) and migration of SCC9 cells (Fig. 3C and D), and 
A

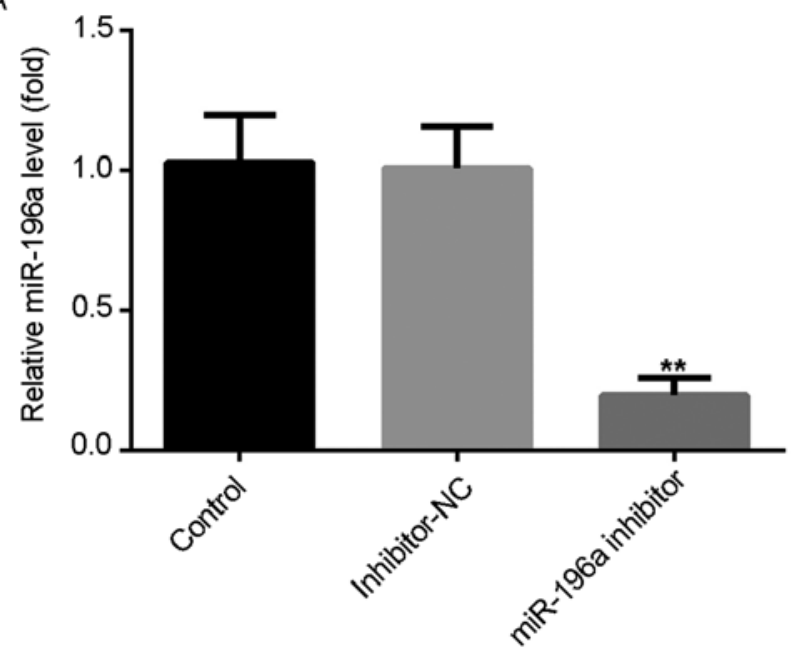

B

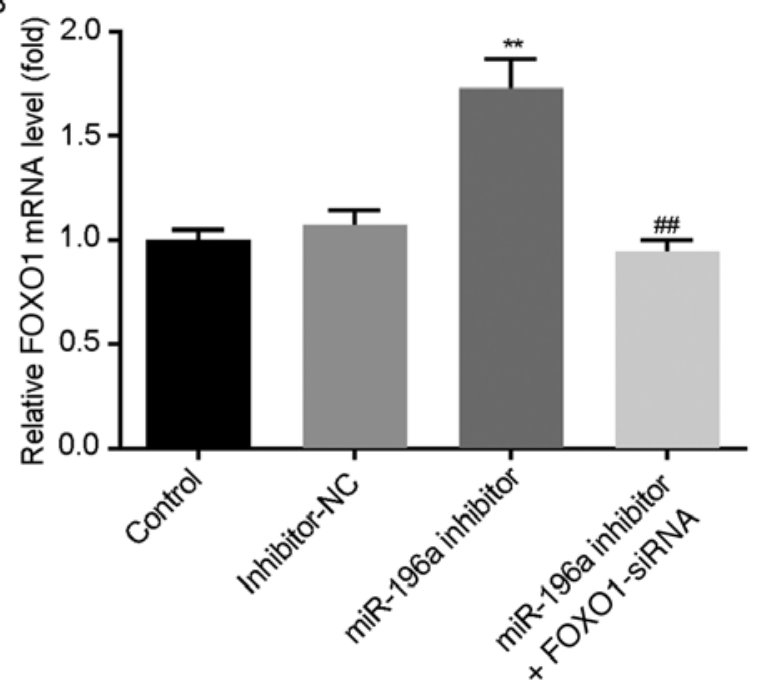

Figure 4. miR-196a regulates human oral squamous cell carcinoma functions by targeting FOXO1. (A) RT-qPCR was performed to detect the relative expression of miR-196a in SCC9 cells transfected with inhibitor-NC or miR-196a inhibitor. (B) The expression of FOXO1 mRNA in SCC9 cells following transfection was detected via RT-qPCR. ${ }^{* *} \mathrm{P}<0.01$ vs. inhibitor-NC group; ${ }^{\# \#} \mathrm{P}<0.01$ vs. miR-196a inhibitor group. RT-qPCR, reverse transcription-quantitative PCR; FOXO1, forkhead box O1; miR, microRNA; NC, negative control.

inhibited cell apoptosis (Fig. 3E). These results indicated that miR-196a might affect SCC9 cell proliferation, migration and apoptosis by regulating FOXO1 expression.

Downregulation of miR-196a resulted in FOXO1 accumulation and FOXO1 depletion had the opposite effect. Whether miR-196a could reduce the expression of FOXO1 was investigated by transfecting SCC9 cells with miR-196a inhibitor, inhibitor-NC or miR-196a inhibitor + FOXO1-siRNA for $48 \mathrm{~h}$. The results revealed that compared with the inhibitor-NC group, the expression of miR-196a in SCC9 cells was significantly downregulated following transfection with miR-196a inhibitor (Fig. 4A). Furthermore, compared to the inhibitor-NC group, miR-196a inhibitor increased the mRNA expression of FOXO1 in SCC9 cells, whereas FOXO1-siRNA had the opposite effect (Fig. 4B). The results indicated that miR-196a negatively regulated FOXO1 expression in SCC9 cells.

miR-196a inhibitor suppresses cell proliferation and induces SCC9 cell apoptosis. To investigate the role of miR-196a in OSCC proliferation, SCC9 cells were transfected with miR-196a inhibitor, inhibitor-NC or miR-196a inhibitor + FOXO1-siRNA for 48 h. As presented in Fig. 5A, transfection of miR-196a inhibitor markedly decreased the proliferation of SCC9 cells, which was subsequently reversed by FOXO1-siRNA. Additionally, the results revealed that apoptosis rate was increased in the miR-196a inhibitor group, while this increase was counteracted by FOXO1-siRNA (Fig. 5B and C). The results indicated that miR-196a may serve a vital role in OSCC proliferation and apoptosis.

Downregulation of miR-196a inhibited the migration and invasion capacity of SCC 9 cells. To determine the potential regulatory role of miR-196a in the migration of OSCC cells, wound healing and transwell invasion assays were performed (Fig. 6A and B). The migration of SCC9 cells in the miR-196a inhibitor group was significantly decreased, which was reversed by FOXO1-siRNA treatment. These results indicated that miR-196a may serve a vital role in the migration of OSCC cells.

The role of miR-196a in the PI3K/Akt pathway. To further determine the function of miR-196a in OSCC cells, western blotting was performed to detect the effect of miR-196a on the expression of PI3K/Akt pathway proteins. After transfection, FOXO1 levels and the ratio of phosphorylated/unphosphorylated PI3K and Akt in SCC9 cells were measured via western blotting. In SCC9 cells, the downregulation of miR-196a significantly reduced the protein expression of $\mathrm{p}$-PI3K and Akt, while the expression of FOXO1 was significantly increased. Additionally, these effects were reversed by FOXO-siRNA (Fig. 7A and B). These data indicated that miR-196a modulated the PI3K/Akt signaling pathway by targeting FOXO1 in OSCC.

miR-196a mimic promotes cell proliferation, induces apoptosis and enhances migration in SCC9 cells. The effect of miR-196a upregulation on SCC9 cells was examined in the current study. SCC9 cells were transfected with mimic control or miR-196a mimic for $48 \mathrm{~h}$, after which RT-qPCR was performed to detect transfection efficiency. The results indicated that miR-196a mimic significantly enhanced miR-196a expression in SCC9 cells (Fig. 8A). Further analysis indicated that miR-196a mimic significantly enhanced SCC9 cell proliferation (Fig. 8B), inhibited cell apoptosis (Fig. 8C) and enhanced migration (Fig. 8D and E).

\section{Discussion}

Cancer cell invasion and metastasis is arguably the primary reason of mortality in patients with OSCC (26). Elucidating the underlying molecular mechanisms of OSCC may therefore allow for the determination of novel treatment strategies for patients with OSCC. Consistent with previous research (27), the present study indicated that miRNA served crucial roles in 
A
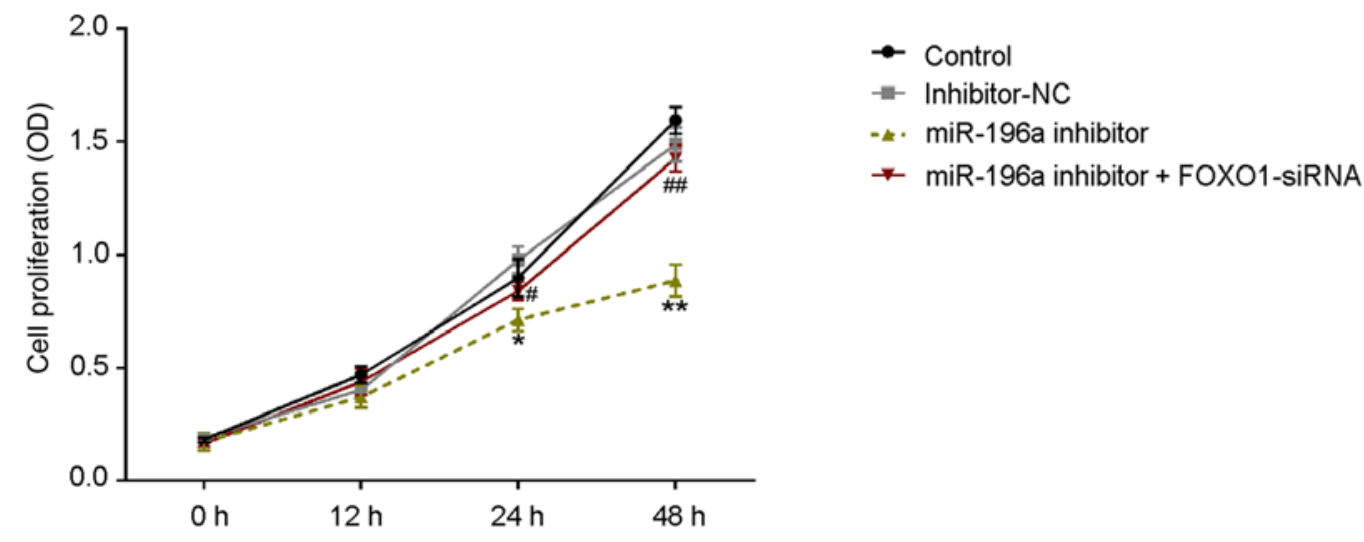

B
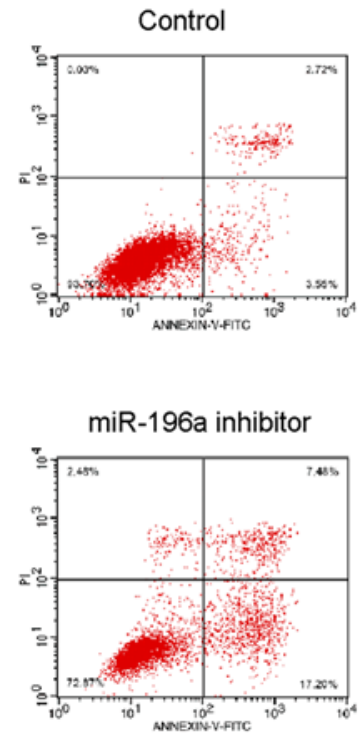
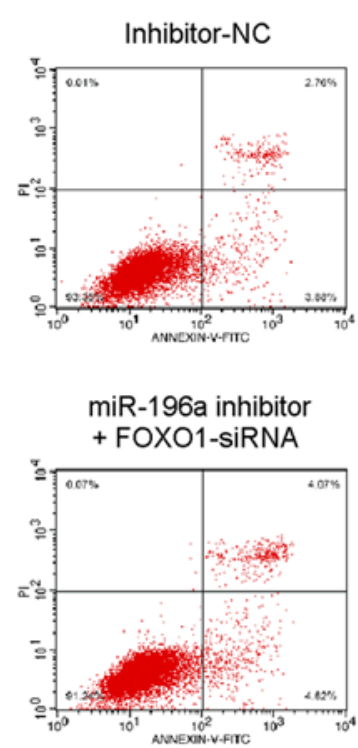

C

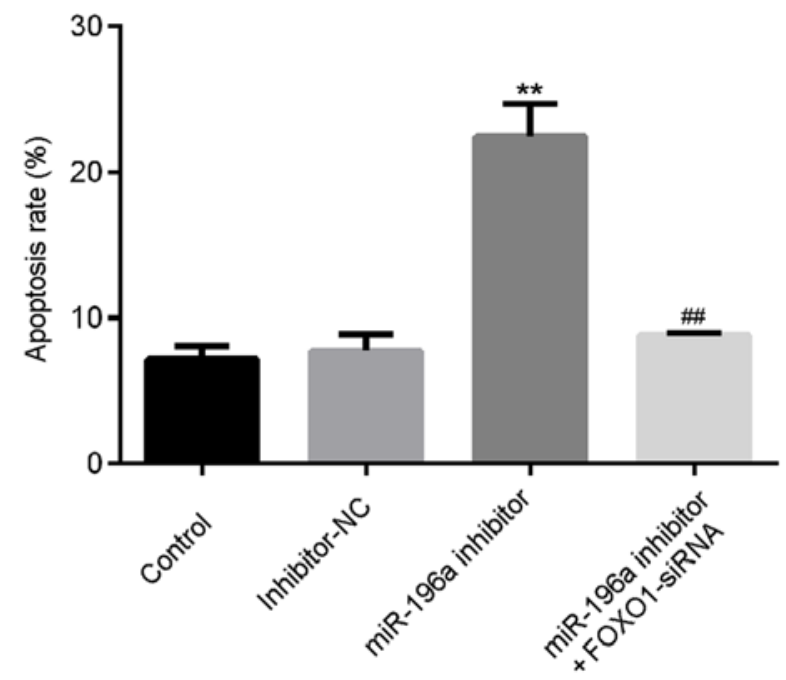

Figure 5. Effects of miR-196a downregulation on SCC9 cell proliferation and apoptosis. SCC9 cells were transfected with miR-196a inhibitor, inhibitor-NC or miR-196a inhibitor + FOXO1-siRNA for $48 \mathrm{~h}$. (A) MTT assays were used to evaluate the role of miR-196a on the proliferation of SCC9 cells. Cell apoptosis was analyzed via flow cytometry. (B) Representative histograms and (C) the rate of apoptosis in SCC9 cells following treatment. data are presented as the mean \pm standard deviation. ${ }^{*} \mathrm{P}<0.05$ and ${ }^{* *} \mathrm{P}<0.01$ vs. inhibitor-NC group; ${ }^{\#} \mathrm{P}<0.05$ and ${ }^{\# \#} \mathrm{P}<0.01$ vs. miR-196a inhibitor group. FOXO1, forkhead box O1; miR, microRNA; NC, negative control; OD, optical density; siRNA, small interfering RNA.

OSCC cell invasion and metastasis. miR-196a acted as a negative regulator of tumor-suppressor genes, promoting cell proliferation and regulating the expression of several genes including FOXO1, p27, IкB $\alpha$, netrin4, mTOR and PI3K/AKT $(28,29)$. However, the biological function and underlying mechanisms of miR-196a in OSCC invasion remain unclear.

The present study assessed whether miR-196a had an effect on the proliferation, apoptosis and migration of OSCC cells by regulating its target gene, FOXO1. The results of the current study indicated that the expression of miR-196a was upregulated in OSCC cells compared with normal HOK cells. miR-196a has been reported to function by targeting FOXO1 in lung cancer, human liver cancer and cervical cancer cells (15-17). In the current study, it was demonstrated that FOXO1 was a direct target of miR-196a. The FOXO1 gene has been demonstrated to mediate several biological processes in cancer cells, including cell proliferation, apoptosis, invasion, migration and angiogenesis (30). The present study also investigated the effect of FOXO1 silencing on OSCC cells. It was determined that FOXO1-siRNA significantly promoted
SCC9 cell proliferation, migration and inhibited SCC9 cell apoptosis. These results indicated that miR-196a might affect SCC9 cell proliferation, migration and apoptosis by regulating FOXO1 expression. The results also revealed that miR-196a negatively mediated the expression of FOXO1 in SCC9 cells. Additionally, the results demonstrated that downregulation of miR-196a inhibited SCC9 cell growth, induced cell apoptosis and decreased cell migration in SCC9 cells. The expression of p-PI3K, p-Akt, PI3K, Akt and FOXO1 were subsequently investigated. It was revealed that $\mathrm{p}$-PI3K and $\mathrm{p}$-Akt were decreased in SCC9 cells following miR-196a downregulation. Thus, it was hypothesized that the mRNA-196a inhibitor may affect OSCC cancer cell processes by preventing PI3K/Akt signaling pathway activation, which may be an important therapeutic strategy for patients with OSCC. All the previously described effects of miR-196a inhibitor on SCC9 cells determined in the present study were reversed following FOXO1 silencing. Furthermore, it was revealed that miR-196a mimic significantly enhanced SCC 9 cell proliferation and migration, and inhibited cell apoptosis. 
A
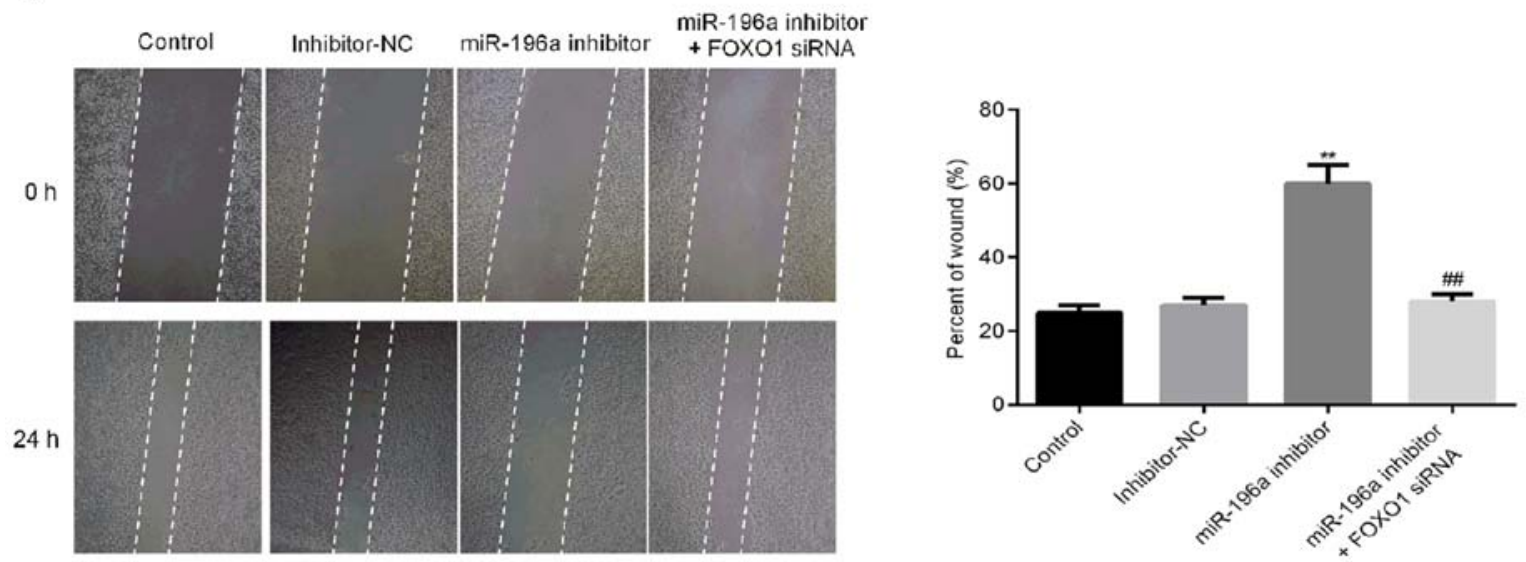

B
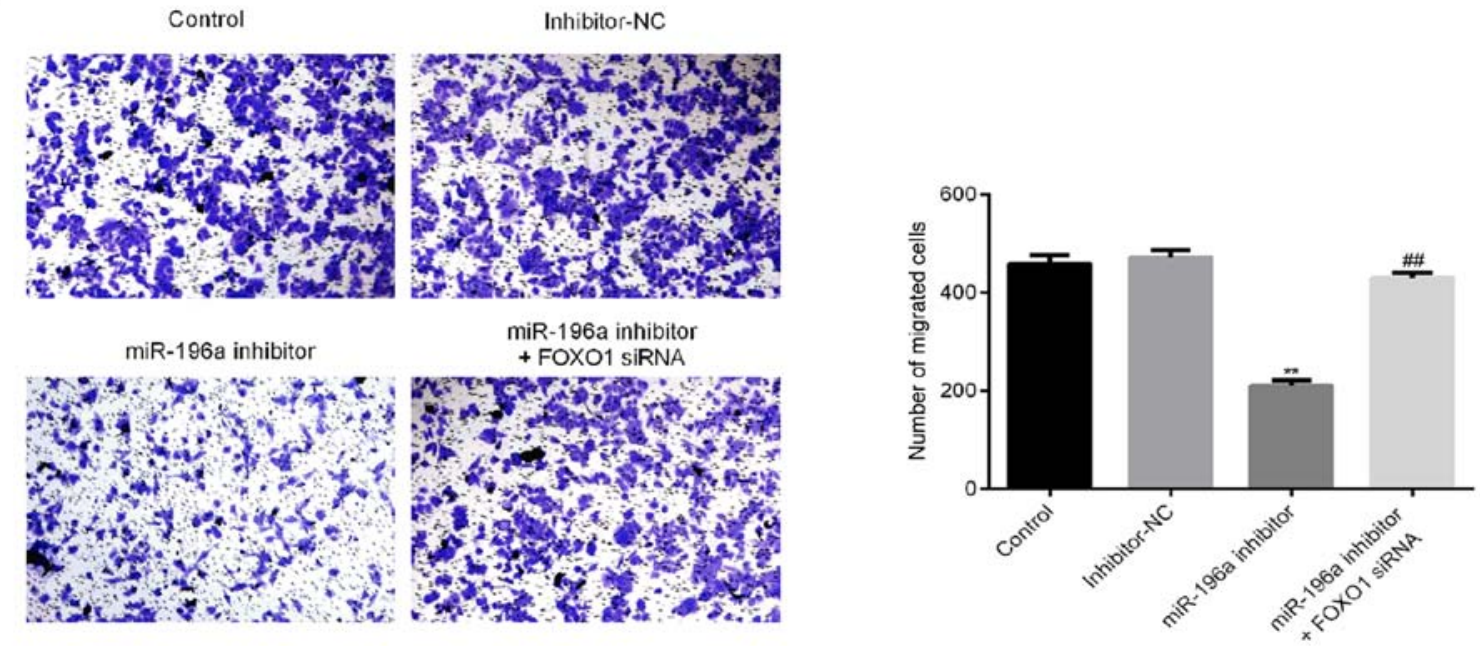

Figure 6. Effects of downregulating miR-196a and FOXO1 on SCC9 cell migration and invasion. SCC9 cells were transfected with miR-196a inhibitor, inhibitor-NC or miR-196a inhibitor + FOXO1-siRNA for $48 \mathrm{~h}$. Cell migration was determined using (A) wound healing assays and (B) transwell assays (magnification, x100). ${ }^{* *} \mathrm{P}<0.01$ vs. inhibitor-NC group; ${ }^{* \#} \mathrm{P}<0.01$ vs. miR-196a inhibitor group. FOXO1, forkhead box O1; miR, microRNA; NC, negative control; siRNA, small interfering RNA.

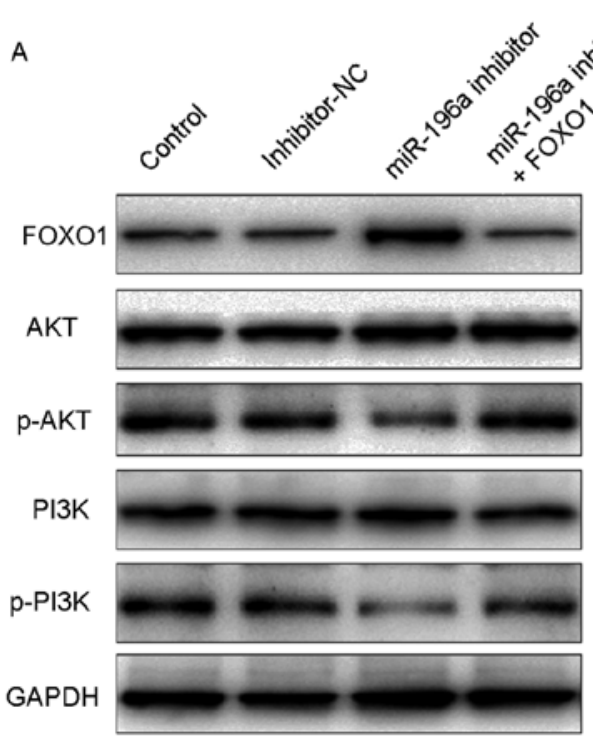
B

Figure 7. Effects of downregulating miR-196a and FOXO1 on the expression of PI3K/Akt signaling pathway proteins. SCC9 cells were transfected with miR-196a inhibitor, inhibitor-NC or miR-196a inhibitor + FOXO1-siRNA for $48 \mathrm{~h}$. Western blot analysis was performed to determine the protein expression of FOXO1, p-PI3K, PI3K, p-Akt and Akt. (A) Representative blots and (B) normalized protein levels are presented. "* $\mathrm{P}<0.01$ vs. inhibitor-NC group; ${ }^{* / P} \mathrm{P}<0.01$ vs. miR-196a inhibitor group. FOXO1, forkhead box O1; miR, microRNA; NC, negative control; p, phosphorylated; siRNA, small interfering RNA. 
A

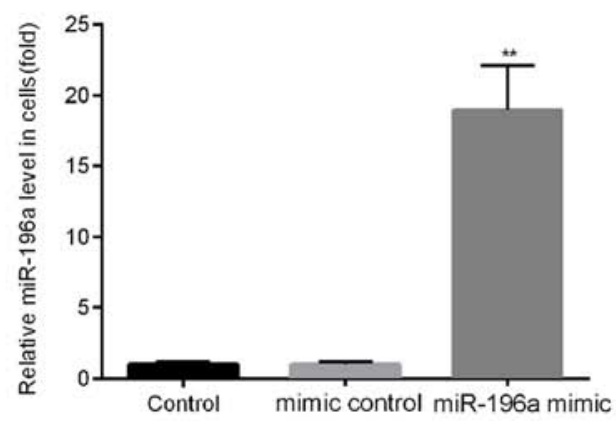

C

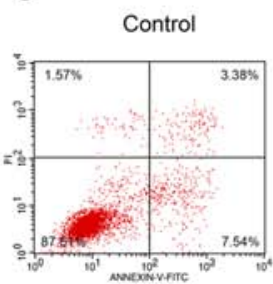

D
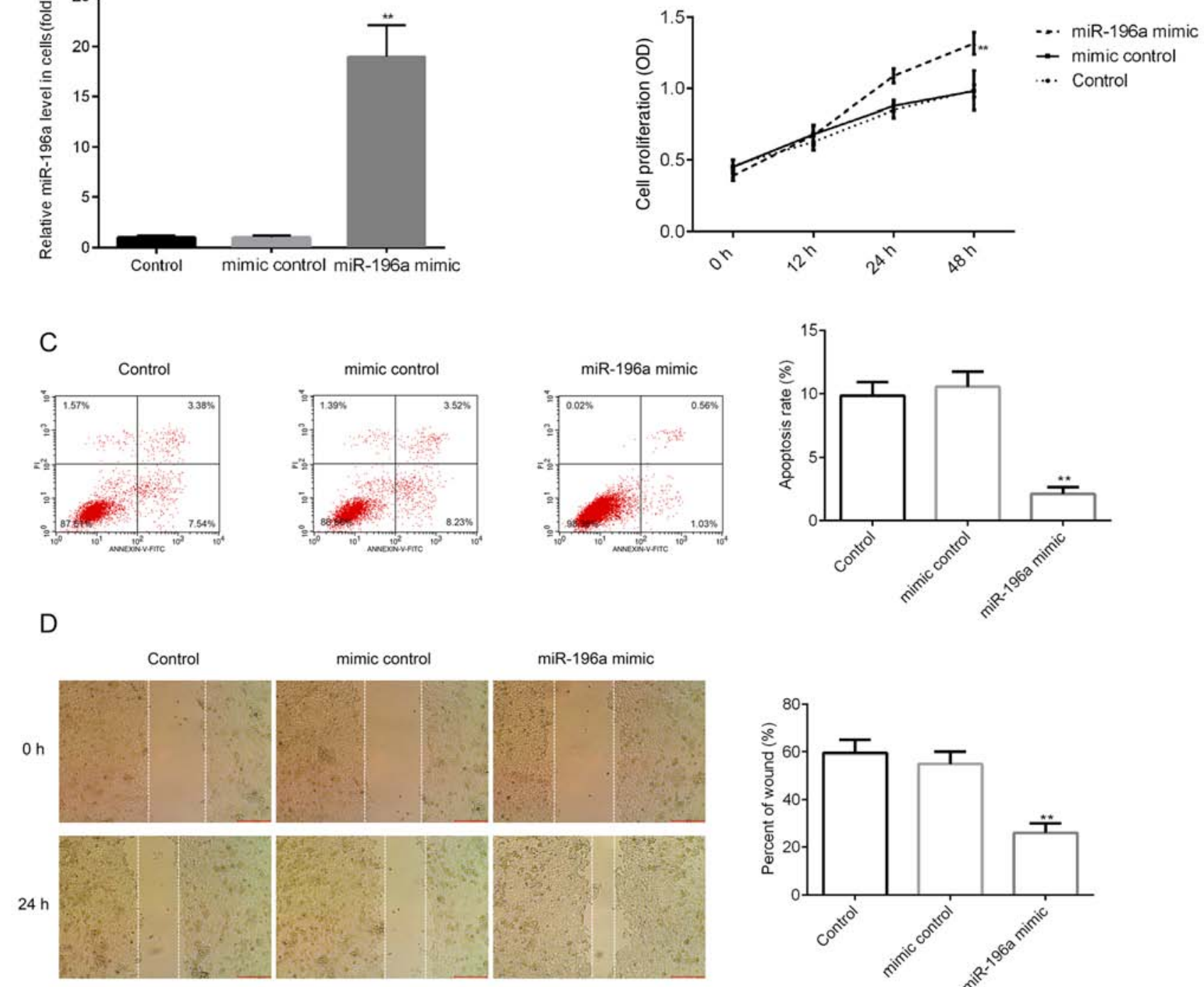

miR-196a mimic
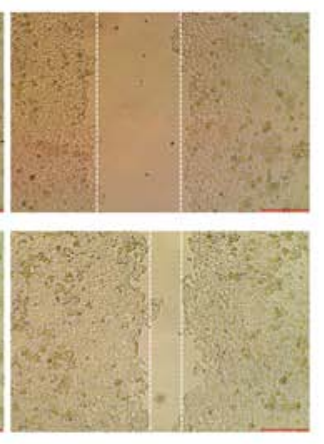

$\mathrm{E}$

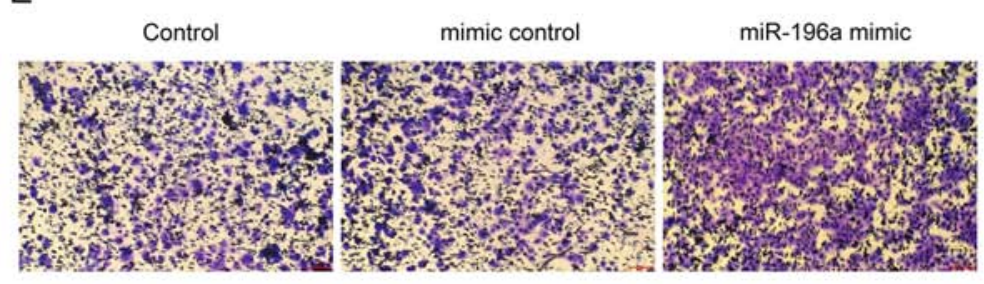

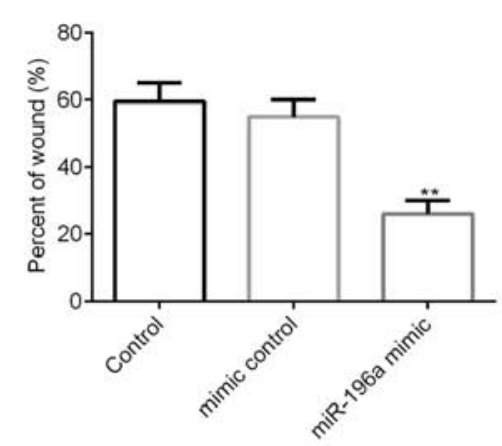

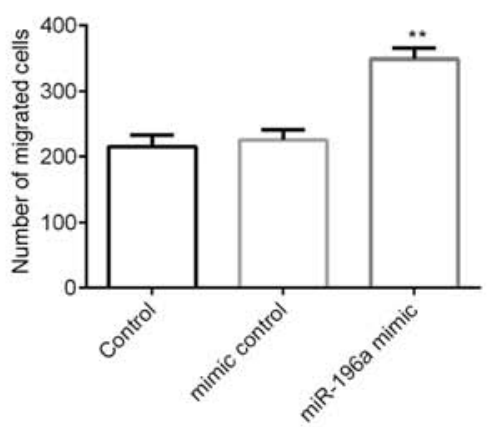

Figure 8. Effect of miR-196a mimic on SCC9 cells. SCC9 cells were transfected with mimic control or miR-196a mimic for 48 h. (A) Reverse transcription-quantitative PCR was performed to detect the level of miR-196a in SCC9 cells. (B) MTT assays were used to detect cell proliferation. (C) Flow cytometry was used to analyze cell apoptosis. Cell migration was determined using (D) wound healing assays and (E) cell transwell assays (magnification, $\mathrm{x} 100) .{ }^{* *} \mathrm{P}<0.01$ vs. inhibitor-NC group. miR, microRNA; OD, optical density.

These data indicated that miR-196a was upregulated in OSCC cells and that the downregulation of miR-196a inhibited OSCC cell proliferation and migration, and induced OSCC cell apoptosis due to its regulation of FOXO1. Therefore, the current resulted indicated a novel therapeutic strategy for OSCC treatment. miR-196a together with FOXO1 are hypothesized to be a potential clinical therapy for treating patients with OSCC. However, the current study is only a preliminary investigation into the role of miR-196a in OSCCs. To demonstrate a more convincing role for miR-196a in OSCC, further in-depth studies need to be performed. For example, the role of miR-196a and FOXO1 in other OSCC cell lines needs to be reviewed. Furthermore, the role of miR-196a and FOXO1 in OSCCs should be investigated in vivo. 


\section{Acknowledgements}

Not applicable.

\section{Funding}

No funding was received.

\section{Availability of data and materials}

The datasets used and/or analyzed during the current study are available from the corresponding author on reasonable request.

\section{Authors' contributions}

HS contributed to study design, data collection, statistical analysis, data interpretation and manuscript preparation. LL, ML, XW and JZ contributed to data collection and statistical analysis. SZ contributed to data collection, statistical analysis and manuscript preparation. All authors read and approved the final manuscript.

\section{Ethics approval and consent to participate}

Not applicable.

\section{Patient consent for publication}

Not applicable.

\section{Competing interests}

The authors declare that they have no competing interests.

\section{References}

1. Curado MP and Hashibe M: Recent changes in the epidemiology of head and neck cancer. Curr Opin Oncol 21: 194-200, 2009.

2. Warnakulasuriya S: Living with oral cancer: Epidemiology with particular reference to prevalence and life-style changes that influence survival. Oral Oncol 46: 407-410, 2010.

3. Mascolo M, Siano M, Ilardi G, Russo D, Merolla F, De Rosa G and Staibano S: Epigenetic disregulation in oral cancer. Int J Mol Sci 13: 2331-2353, 2012.

4. Sharma A, Mendez E, Yueh B, Lohavanichbutr P, Houck J, Doody DR, Futran ND, Upton MP, Schwartz SM and Chen C: Human papillomavirus-positive oral cavity and oropharyngeal cancer patients do not have better quality-of-life trajectories. Otolaryngol Head Neck Surg 146: 739-745, 2012.

5. Reid A, Garrett E, Dibben C and Williamson L: 'A confession of ignorance': Deaths from old age and deciphering cause-of-death statistics in Scotland, 1855-1949. Hist Fam 20: 320-344, 2015.

6. Wang Z, Cai H, Lin L, Tang M and Cai H: Upregulated expression of microRNA-214 is linked to tumor progression and adverse prognosis in pediatric osteosarcoma. Pediatr Blood Cancer 61: 206-210, 2014.

7. Farh KK, Grimson A, Jan C, Lewis BP, Johnston WK, Lim LP, Burge CB and Bartel DP: The widespread impact of mammalian MicroRNAs on mRNA repression and evolution. Science 310: 1817-1821, 2005.

8. Nohata N, Hanazawa T, Kinoshita T, Okamoto Y and Seki N: MicroRNAs function as tumor suppressors or oncogenes: Aberrant expression of microRNAs in head and neck squamous cell carcinoma. Auris Nasus Larynx 40: 143-149, 2013.

9. Lamouille S, Subramanyam D, Blelloch R and Derynck R: Regulation of epithelial-mesenchymal and mesenchymal-epithelial transitions by microRNAs. Curr Opin Cell Biol 25: 200-207, 2013.
10. Ell B and Kang Y: MicroRNAs as regulators of bone homeostasis and bone metastasis. Bonekey Rep 3: 549, 2014.

11. Ge J, Chen Z, Li R, Lu T and Xiao G: Upregulation of microRNA-196a and microRNA-196b cooperatively correlate with aggressive progression and unfavorable prognosis in patients with colorectal cancer. Cancer Cell Int 14: 128, 2014.

12. Liu XH, Lu KH, Wang KM, Sun M, Zhang EB, Yang JS, Yin DD, Liu ZL, Zhou J, Liu ZJ, et al: MicroRNA-196a promotes non-small cell lung cancer cell proliferation and invasion through targeting HOXA5. BMC Cancer 12: 348, 2012.

13. Tsai MM, Wang CS, Tsai CY, Chen CY, Chi HC, Tseng YH, Chung PJ, Lin YH, Chung IH, Chen CY and Lin KH: MicroRNA-196a/-196b promote cell metastasis via negative regulation of radixin in human gastric cancer. Cancer Lett 351: 222-231, 2014.

14. Zhang C, Yao C, Li H, Wang G and He X: Combined elevation of microRNA-196a and microRNA-196b in sera predicts unfavorable prognosis in patients with osteosarcomas. Int J Mol Sci 15: 6544-6555, 2014

15. Guerriero I, D'Angelo D, Pallante P, Santos M, Scrima M, Malanga D, De Marco C, Ravo M, Weisz A, Laudanna, et al: Analysis of miRNA profiles identified miR-196a asa crucial mediator of aberrant PI3K/AKT ignaling in lung cancer cells. Oncotarget 8: 19172-19191, 2017.

16. Yang L, Peng F, Qin J, Zhou H and Wang B: Downregulation of microRNA-196a inhibits human liver cancer cell proliferation and invasion by targeting FOXO1. Oncol Rep 38: 2148-2154, 2017.

17. Hou T, Ou J, Zhao X, Huang X, Huang Y and Zhang Y: MicroRNA-196a promotes cervical cancer proliferation through the regulation of FOXO1 and p27Kip1. Br J Cancer 110: 1260-1268, 2014.

18. Guo H, German P, Bai S, Barnes S, Guo W, Qi X, Lou H, Liang J, Jonasch E, Mills GB and Ding Z: The PI3K/AKT pathway and renal cell carcinoma. J Genet Genomics 42: 343-353, 2015.

19. Gao Y, Ma C, Feng X, Liu Y and Haimiti X: BF12, a novel benzofuran, exhibits anti-tumor activity by inhibiting microtubules and the PI3K/Akt/mTOR signaling pathway in human cervical cancer cells. Chem Biodivers: Jan 17, 2020 (Epub ahead of print).

20. Sobočan M, Bračič S, Knez J, Takač I and Haybaeck J: The Communication Between the PI3K/AKT/mTOR pathway and Y-box binding protein-1 in gynecological cancer. Cancers (Basel) 12: E205, 2020.

21. An J, He H, Yao W, Shang Y, Jiang Y and Yu Z: PI3K/Akt/FoxO pathway mediates glycolytic metabolism in HepG2 cells exposed to triclosan (TCS). Environ Int 136: 105428, 2020.

22. Nozhat Z, Mohammadi-Yeganeh S, Azizi F, Zarkesh M and Hedayati M: Effects of metformin on the PI3K/AKT/FOXO1 pathway in anaplastic thyroid Cancer cell lines. Daru 26: 93-103, 2018.

23. Zheng M, Cao MX, Yu XH, Li L, Wang K, Wang SS, Wang HF, Tang YJ, Tang YL and Liang XH: STAT3 promotes invasion and aerobic glycolysis of human oral squamous cell carcinoma via inhibiting FoxO1. Front Oncol 9: 1175, 2019.

24. Gao C, Ren C, Liu Z, Zhang L, Tang R and Li X: GAS5, a FoxO1-actived long noncoding RNA, promotes propofol-induced oral squamous cell carcinoma apoptosis by regulating the miR-1297-GSK3 $\beta$ axis. Artif Cells Nanomed Biotechnol 47: 3985-3993, 2019.

25. Livak KJ and Schmittgen TD: Analysis of relative gene expression data using real-time quantitative PCR and the 2(-Delta Delta C(T)) method. Methods 25: 402-408, 2001

26. Steeg PS: Tumor metastasis: Mechanistic insights and clinical challenges. Nat Med 12: 895-904, 2006.

27. Sharma M, Sah P, Sharma SS and Radhakrishnan R: Molecular changes in invasive front of oral cancer. J Oral Maxillofac Pathol 17: 240-247, 2013.

28. Huang WC, Chan SH, Jang TH, Chang JW, Ko YC, Yen TC, Chiang SL, Chiang WF, Shieh TY, Liao CT, et al: MiRNA-491-5p and GIT1 serve as modulators and biomarkers for oral squamous cell carcinoma invasion and metastasis. Cancer Res 74: 751-764, 2014.

29. Nagai H, Hasegawa S, Uchida F, Terabe T, Ishibashi Kanno N, Kato K, Yamagata K, Sakai S, Kawashiri S, Sato H, et al: MicroRNA-205-5p suppresses the invasiveness of oral squamous cell carcinoma by inhibiting TIMP2 expression. Int J Oncol 52: 841-850, 2018.

30. Feng X, Luo Q, Wang H, Zhang H and Chen F: MicroRNA-22 suppresses cell proliferation, migration and invasion in oral squamous cell carcinoma by targeting NLRP3. J Cell Physiol 233: 6705-6713, 2018.

This work is licensed under a Creative Commons Attribution-NonCommercial-NoDerivatives 4.0 International (CC BY-NC-ND 4.0) License. 